\title{
Lysosomal damage after spinal cord injury causes accumulation of RIPK1 and RIPK3 proteins and potentiation of necroptosis
}

\author{
Shuo Liu ${ }^{1,2}$, Yun Li', Harry M. C. Choi ${ }^{1}{ }^{1}$, Chinmoy Sarkar ${ }^{1}$, Eugene Y. Koh², Junfang Wu ${ }^{1}$ and Marta M. Lipinski ${ }^{1}$
}

\begin{abstract}
Necroptosis, a regulated necrosis pathway mediated by the receptor-interacting protein kinases 1 and 3 (RIPK1 and RIPK3), is induced following spinal cord injury (SCl) and thought to contribute to neuronal and glial cell death. However, mechanisms leading to activation of necroptosis after $\mathrm{SCl}$ remain unclear. We have previously shown that autophagy, a catabolic pathway facilitating degradation of cytoplasmic proteins and organelles in a lysosomedependent manner, is inhibited following SCl in rats. Our current data confirm that inhibition of autophagy also occurs after thoracic contusive $\mathrm{SCl}$ in the mouse model, as indicated by accumulation of both the autophagosome marker, LC3-II and autophagy cargo protein, p62/SQSTM1. This was most pronounced in the ventral horn neurons and was caused by rapid inhibition of lysosomal function after SCI. Interestingly, RIPK1, RIPK3, and the necroptosis effector protein MLKL also rapidly accumulated after $\mathrm{SCl}$ and localized to neurons with disrupted autophagy, suggesting that these events may be related. To determine if lysosomal dysfunction could contribute to induction of necroptosis, we treated PC12 cells and primary rat cortical neurons with lysosomal inhibitors. This led to rapid accumulation of RIPK1 and RIPK3, confirming that they are normally degraded by the lysosomal pathway. In PC12 cells lysosomal inhibition also sensitized cells to necroptosis induced by tumor necrosis factor a (TNFa) and caspase inhibitor. Imaging studies confirmed that RIPK1 partially localized to lysosomes in both untreated and lysosomal inhibitor treated cells. Similarly, we detected presence of RIPK1, RIPK3 and MLKL in both cytosol and at lysosomes after SCI in vivo. Furthermore, stimulation of autophagy and lysosomal function with rapamycin treatment led to decreased accumulation of RIPK1 and attenuated cell death after SCl. These data suggest that lysosomal dysfunction after SCl may contribute to both inhibition of autophagy and sensitize cells to necroptosis by promoting RIPK1 and RIPK3 accumulation.
\end{abstract}

\section{Introduction}

One of the features of secondary injury following traumatic spinal cord injury (SCI) is progressive neuronal cell death. The mechanisms of this death are diverse and include necrosis, classical apoptosis, as well as caspaseindependent regulated cell death pathways ${ }^{1}$. Because

\footnotetext{
Correspondence: Junfang Wu (junfang.wu@som.umaryland.edu) or Marta M. Lipinski (mlipinski@som.umaryland.edu)

${ }^{1}$ Department of Anesthesiology and the Center for Shock, Trauma and Anesthesiology Research (STAR), University of Maryland School of Medicine, Baltimore, MD, USA

²Department of Orthopaedics, University of Maryland School of Medicine, Baltimore, MD, USA

Edited by A. Verkhratsky
}

many pathways are involved, attempts at improving SCI outcomes by inhibiting specific types of cell death have been largely unsuccessful ${ }^{2}$. This has led to more recent proposals for use of drugs that target upstream changes involved in the initiation of multiple cell death pathways. This, however, will require identification and characterization of the upstream cellular events involved in control of multiple pro-death pathways during SCI secondary injury.

Necroptosis is a form of regulated necrosis activated downstream of the tumor necrosis factor receptor 1 (TNFR1), dependent on the activity of the receptorinteracting protein kinase 1 (RIPK1) and 3 (RIPK3) and

\section{(c) The Author(s) 2018}

(c) (i) Open Access This article is licensed under a Creative Commons Attribution 4.0 International License, which permits use, sharing, adaptation, distribution and reproduction in any medium or format, as long as you give appropriate credit to the original author(s) and the source, provide a link to the Creative Commons license, and indicate if changes were made. The images or other third party material in this article are included in the article's Creative Commons license, unless indicated otherwise in a credit line to the material. If material is not included in the article's Creative Commons license and your intended use is not permitted by statutory regulation or exceeds the permitted use, you will need to obtain permission directly from the copyright holder. To view a copy of this license, visit http://creativecommons.org/licenses/by/4.0/. 
mediated by the mixed lineage pseudo-kinase MLKL ${ }^{3,4}$. The upstream mediator of necroptosis, RIPK1, is a key regulator of the innate immune responses involved in both inflammation and cell death, thus may represent an ideal target for reducing both cell death and inflammation in the central nervous system $(\mathrm{CNS})^{5}$. Activation of necroptosis has been shown to contribute to cell loss and tissue damage in neurodegenerative diseases affecting the spinal cord, such as amyotrophic lateral sclerosis and multiple sclerosis ${ }^{6,7}$. Recent data also demonstrate involvement of necroptosis in neuronal and glial cell death after $\mathrm{SCI}^{8-10}$, and that its inhibition can improve functional recovery in animal models of $\mathrm{SCI}^{10,11}$. However, the mechanisms leading to activation of necroptosis after SCI and its relationship to other cellular pathways in this context remain unknown.

We recently demonstrated that contusive SCI in a rat model leads to inhibition of autophagy, a lysosomedependent protein degradation pathway ${ }^{12}$. Inhibition of autophagy after SCI is particularly pronounced in the ventral horn motor neurons and contributes to endoplasmic reticulum (ER) stress induced apoptosis in these cells. Here we demonstrate that autophagy is similarly inhibited after SCI in a mouse model. Inhibition of autophagy flux is caused by a rapid decline in lysosomal function after injury, leading to accumulation of dysfunctional autophagosomes. Surprisingly, our data demonstrate that inhibition of lysosomal function also causes rapid accumulation of necroptosis mediators, RIPK1, RIPK3, and MLKL in neurons both in vitro and in vivo after SCI. These proteins accumulate both in the cytosol and at the lysosomes and can lead to cellular sensitization to necroptosis. Conversely, increasing function of the autophagy-lysosomal pathway in vivo can decrease necroptosis and general cell damage after SCI. These data point to a previously unexplored link between inhibition of the autophagy-lysosomal pathway and induction of neuronal necroptosis after SCI, and suggest improving lysosomal function as a potential multifunctional treatment after SCI.

\section{Results}

$\mathrm{SCl}$ leads to lysosomal dysfunction and inhibition of autophagy flux in mice

We previously demonstrated that SCI leads to a temporary inhibition of autophagy in the rat contusion model $^{12}$. To determine whether this is also the case in mice, we assessed accumulation of autophagosomes and autophagy flux after thoracic (T10) contusive injury ${ }^{13}$. Western blot data demonstrated progressive accumulation of the autophagy marker microtubule-associated protein 1A/1B light chain 3-II (LC3-II) in the spinal cord tissue surrounding the injury site starting by $6 \mathrm{~h}$ (Fig. 1a, b and Supplementary Figure S1a). This was accompanied by rapid accumulation of $\mathrm{p} 62 / \mathrm{SQSTM} 1$ protein starting at 1 $\mathrm{h}$ after injury, indicating inhibition of autophagy flux ${ }^{14}$ (Fig. 1a, c). Interestingly, while increased LC3-II levels persisted up to 1 month after injury, levels of p62/ SQSTM1 substantially declined within 1 week, indicating partial restoration of autophagy flux at later time points after SCI. These data were confirmed by immunohistochemistry (IHC) in GFP-LC3 transgenic autophagy reporter mice ${ }^{15,16}$, demonstrating accumulation of GFPLC3 and p62/SQSTM1 positive cells in injured spinal cord tissues (Fig. 1d, e). Consistent with the western blot data, GFP-LC3 positive cells persisted, while p62/ SQSTM1 staining declined by 1 week after injury (Fig. 1e and Supplementary Figure S2). High-magnification imaging confirmed that GFP-LC3 localized to intracellular puncta corresponding to autophagosomes (Fig. If, g). Similar data were obtained by IHC staining with LC3 antibody in wild type mice (Fig. $1 \mathrm{~h}$ ). As in rat $\mathrm{SCI}^{12}$, the initial accumulation of autophagosomes and inhibition of autophagy flux at day 1 after SCI occurred primarily in neurons (Fig. 1h, i), with motor neurons of the ventral horn affected to the highest degree.

To determine the mechanisms leading to inhibition of autophagy flux we assessed lysosomal function after SCI. Consistent with previous reports ${ }^{17}$, levels of the lysosomal enzyme cathepsin D (CTSD) dramatically increased at late time points after injury (days 3 and 7). This reflects activation and proliferation of microglia and infiltrating macrophages after injury (data not shown $)^{12,16,18}$. However, at early time points ( 1 and $6 \mathrm{~h}$ ) after SCI we observed a slight decrease in levels of CTSD, indicating possible injury-induced decrease in lysosomal function (Fig. 2a, b and Supplementary Figure S1b). This is consistent with our previous rat data demonstrating defects in autophagosome-lysosome fusion after $\mathrm{SCI}^{12}$. To further investigate the initial decline in lysosomal function after $\mathrm{SCI}$, we purified lysosome-enriched fractions ${ }^{16}$ from sham control and injured spinal cords at 2 and $24 \mathrm{~h}$. We observed decrease in both precursor and cleaved CTSD protein levels at the lysosomes (Fig. 2c, d and Supplementary Figure S3a). Furthermore, enzymatic activity assay confirmed decreased CTSD activity at 6 and $24 \mathrm{~h}$ after SCI (Fig. 2e, f). Similar decline was observed for another lysosomal enzyme, $\mathrm{N}$-acetyl-alphaglucosaminidase (NAG) (Fig. 2e, f), consistent with our hypothesis that SCI leads to rapid decrease in lysosomal function, thus causing inhibition of autophagy flux.

\section{Autophagy/lysosomal damage correlates with markers of necroptosis in the injured spinal cord}

We previously demonstrated that inhibition of autophagy/lysosomal function after SCI leads to exacerbation of ER stress and contributes to neuronal apoptosis ${ }^{12}$. However, non-apoptotic cell death pathways are thought to 


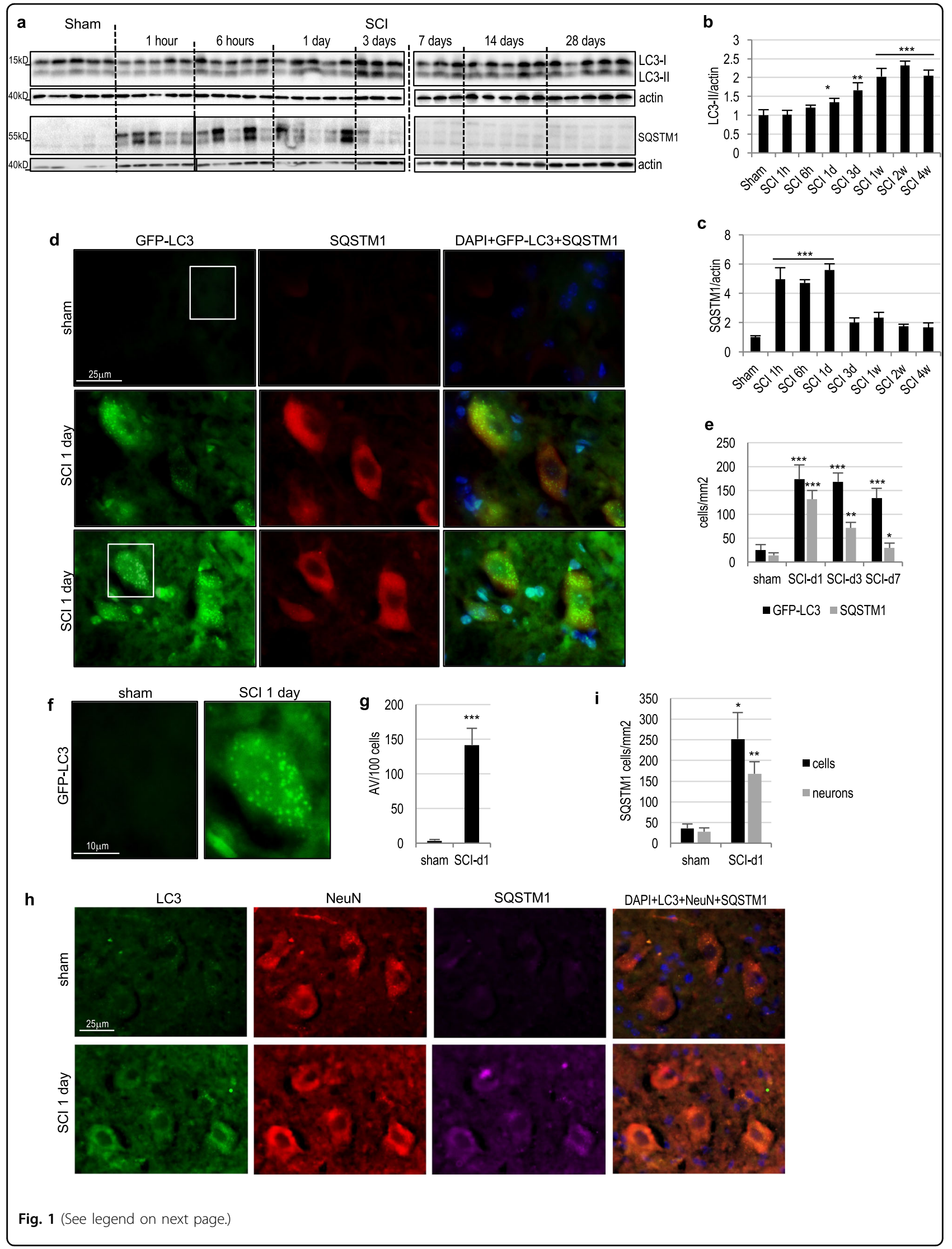


(see figure on previous page)

Fig. $1 \mathrm{SCl}$ leads to disruption of autophagy flux in ventral horn motor neurons. a Time course of accumulation of LC3-II and p62/SQSTM1 proteins in the spinal cord tissue surrounding injury site following thoracic contusion SCl in a mouse model. Each blot lane represents an individual animal. Full unedited western blots are presented in Supplemental Figure S1a. b Quantification of LC3-II levels from a and S1a. c Quantification of p62/SQSTM1 levels from a and S1a. $n=5$. $\mathbf{d}$ IHC staining demonstrating accumulation of the GFP-LC3 autophagosome marker and p62/SQSTM1 in the same ventral horn cells $24 \mathrm{~h}$ after SCl in GFP-LC3 transgenic autophagy reporter mice. Images were acquired at $60 \times$ magnification. Images for day 3 and 7 are presented in Supplemental Figure S2. e Quantification of GFP-LC3 and SQSTM1 data in d and S2a. $n=4$. $\mathbf{f}$ Close-up of GFP-LC3 images from areas indicated in d showing accumulation of autophagosomes. $\mathbf{g}$ Quantification of autophagosomes/autophagic vesicles (AV) from data in $\mathrm{d}$ and f. $n=4$. $\mathbf{h} \| \mathrm{HC}$ images demonstrating that GFP-LC3 and p62/SQSTM1 accumulate primarily in neurons in the ventral horn at day 1 after $\mathrm{SCl}$. Neurons were identified by staining with NeuN/RBFOX3. Images were acquired at $\times 20$ magnification. $\mathbf{i}$ Quantification of data from $\mathrm{h}$. $n=4$ All data are presented as mean \pm SE. ${ }^{*} p<0.05,{ }^{* *} p<0.01,{ }^{* * *} p<0.001$

also contribute to secondary injury after $\mathrm{SCI}^{2}$. To specifically assess contribution of necroptosis, we investigated expression of its upstream mediator RIPK $1^{3,4}$. We observed rapid accumulation of RIPK1 in the spinal cord starting $1 \mathrm{~h}$ after SCI (Fig. 3a, b and Supplementary Figure S1c). This is consistent with the timing of lysosomal/ autophagy inhibition and accumulation of p62/SQSTM1. Accumulation of RIPK1 protein was further confirmed by IHC (Fig. 3c, d and Supplementary Figure S4a), which also demonstrated that at 1day after SCI RIPK1 preferentially accumulated in neurons with inhibited autophagy/lysosomal function (p62/SQSTM1 positive, Fig. 3c, e). Therefore, accumulation of the essential necroptosis mediator RIPK 1 occurs at the same time and in the same neuronal cells as SCI-induced defects in lysosomal function. Expression of RIPK1 protein increased further later after injury (days 3 and 7, Fig. 3a, b, d and Supplementary Figure S4a) but at these time points it was mainly localized to microglia and macrophages in the area surrounding injury site (data not shown), suggesting proinflammatory function ${ }^{5}$.

In addition to necroptosis RIPK1 is known to play a role in activation of NFK-B and induction of inflammation, and in regulation of extrinsic apoptosis through caspase $8^{5}$. During necroptosis but not apoptosis or inflammation RIPK1 kinase is activated. This leads to recruitment and activation of necroptosis mediator RIPK3 and recruitment of the necroptosis executor, the mixed lineage pseudokinase, MLKL ${ }^{19}$. By $1 \mathrm{~h}$ after SCI we observed increase in RIPK3 and MLKL protein levels in the spinal cord (Fig. 4a-c and Supplementary Figure S5). Unlike RIPK1, levels of both proteins initially increased rapidly but declined by day 3 after injury, as expected for their function in cell death, which peaks around day 1 after SCI, rather than in later inflammation. The timing of RIPK3 and MLKL accumulation was further confirmed by IHC (Fig. 4d, e, g, h and Supplementary Figure S4b-c). To an extent even higher than RIPK1, RIPK3, and MLKL specifically accumulated in neurons with elevated p62/ SQSTM1 protein (Fig. 4d, f, g, i). Therefore, lysosomal damage after SCI is associated with accumulation of RIPK1, RIPK3, and MLKL, suggesting potential link between lysosomal dysfunction and regulation of necrosome components in neurons.

\section{RIPK1/RIPK3 proteins are regulated by lysosomal degradation}

RIPK1 protein is regulated by ubiquitination, which affects both its function and stability ${ }^{5}$. RIPK1 protein stability has been thought to be regulated mainly by proteasomal degradation. However, a recent report indicated that under at least some circumstances both RIPK1 and RIPK3 may also be degraded via lysosome-dependent pathway $^{20}$. To determine if lysosomal degradation may be important for RIPK1/RIPK3 expression in neural cells, we treated rat PC12 cells with lysosomal inhibitor, chloroquine $(\mathrm{Chq})^{14}$. Four hour treatment led to inhibition of lysosomal function and autophagy flux and accumulation of both RIPK1 and RIPK3 proteins (Fig. 5a, b and Supplementary Figure S6a-b). Similar results were obtained when bafilomycin A (BafA) was used to inhibit lysosomal function (Supplementary Figure S6a, c). RIPK1 and RIPK3 also accumulated in primary rat cortical neurons treated with either Chq or BafA (Fig. 5c-e and Supplementary Figure S6d). Accumulation of RIPK1 and RIPK3 in Chq treated neurons was further confirmed by immunofluorescence (IF) staining (Fig. 5f-h and Supplementary Figure S6e). Image analysis also revealed partial co-localization of RIPK1 protein to LAMP1-positive lysosomes in both Chq treated and untreated cells (Fig. 5i, j). The degree of colocalization increased following Chq treatment indicating specific accumulation of RIPK1 in lysosomes (Fig. 5i, j). These data demonstrate that lysosomal localization and degradation may be involved in normal homeostasis of RIPK1 and RIPK3 proteins in neurons and that lysosomal dysfunction can lead to their accumulation.

\section{Lysosomal inhibition can sensitize cells to necroptosis}

The fact that RIPK1 and RIPK3 are both regulated by lysosomal degradation, suggests that lysosomal function may also affect cellular sensitivity to necroptosis. To test this hypothesis, we treated PC12 cells with rat TNF $\alpha$ in the presence of pan-caspase inhibitor Boc-D to induce 


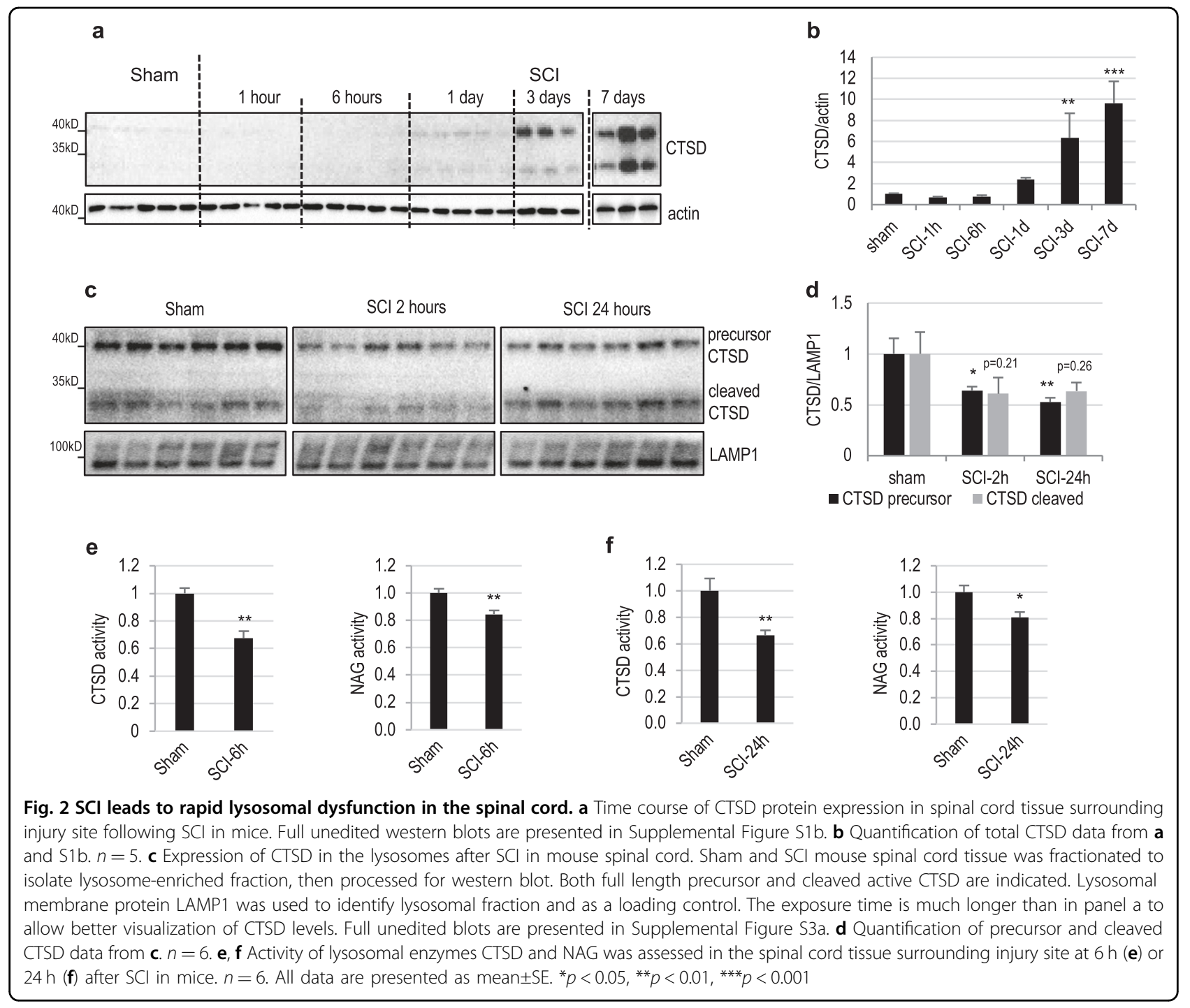

necroptosis. This led to dose-dependent cell death, which was potentiated in the presence of lysosomal inhibitor BafA and suppressed by necroptosis inhibitor, necrostatin $1^{3}$ (Nec1, Fig. 5k, 1 and Supplementary Figure S7). Interestingly, in the presence of BafA caspase inhibition alone was able to induce necroptotic cell death, even without TNF $\alpha$ treatment. This suggests that accumulation of RIPK1/3 upon lysosomal inhibition may be sufficient for necrosome formation and activation of necroptosis.

\section{Necroptosis mediators are present at the lysosomes in spinal cord in vivo}

To confirm that lysosomal dysfunction may be also contributing to regulation of necroptosis in vivo after SCI, we investigated intracellular localization of its mediators. Subcellular fractionation of spinal cord samples revealed that RIPK1, RIPK3, and MLKL were present and accumulated in both cytosolic and lysosome-enriched fractions of the spinal cord after SCI (Fig. 6a-d and Figure S3b), consistent with the hypothesis that they are regulated by lysosomal degradation and accumulate at lysosomes when degradation is blocked.

\section{Restoration of autophagy-lysosomal function attenuates necroptosis after $\mathrm{SCl}$}

To determine if improving autophagy-lysosomal function may decrease induction of necroptosis after SCI, we treated sham and injured mice with mTOR inhibitor, rapamycin, which has been previously shown to improve functional outcomes after $\mathrm{SCI}^{21,22}$. Inhibition of the mTOR pathway is known to both increase autophagy flux and induce lysosomal biogenesis, and we have previously demonstrated that it leads to increase in the number of lysosomes in vivo in the $\operatorname{brain}^{16}$. Similar to previous reports in experimental traumatic brain injury (TBI) ${ }^{23}$, we observed increased phosphorylation of mTOR target, the 
a

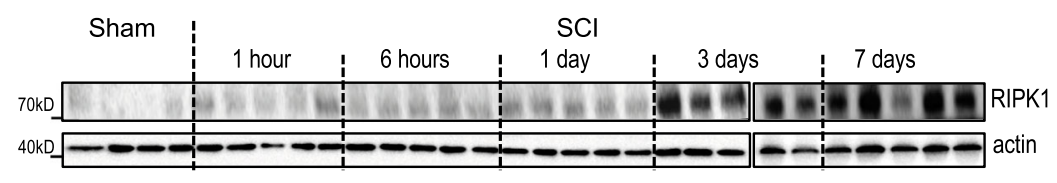

C
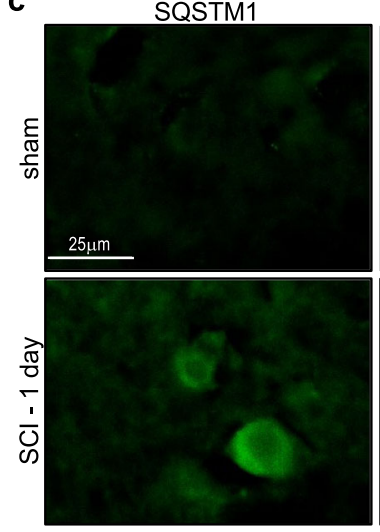
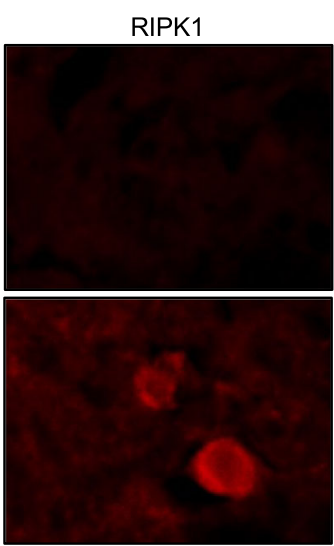

DAPI+SQSTM1+RIPK1

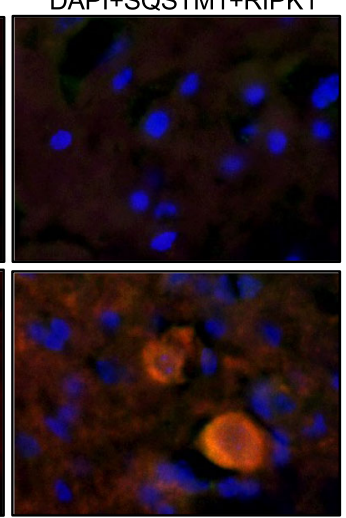

b
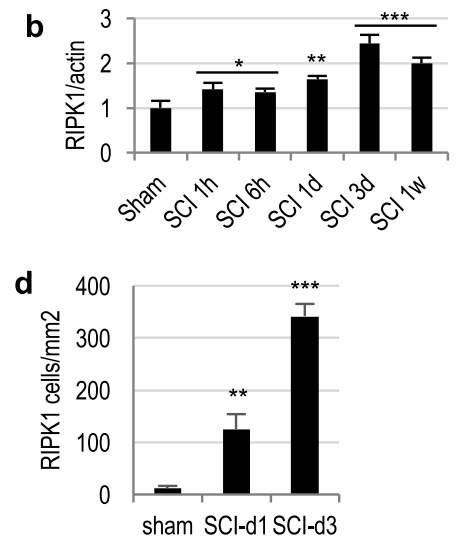

e

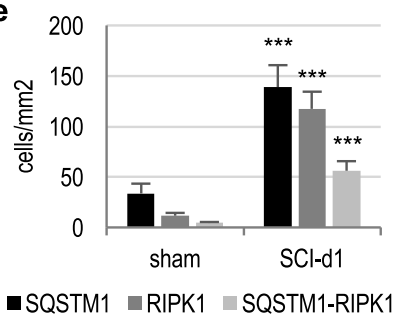

Fig. 3 Expression of the necroptosis regulator RIPK1 correlates with inhibition of autophagy and lysosomal function after SCl. a The time course of RIPK1 protein expression in spinal cord tissue surrounding injury site following $\mathrm{SCl}$ in mice. Full unedited western blots are presented in Supplemental Figure S1c. b Quantification of RIPK1 data from $\mathbf{a}$ and S1C. $n=5$. $\mathbf{c}$ HC staining demonstrates accumulation of RIPK1 and p62/SQSTM1 in the same ventral horn cells at $24 \mathrm{~h}$ after SCl in mice. Images were acquired at $\times 20$ magnification. Full time course images for RIPK1 are presented in Supplemental Figure S4a. d Quantification of RIPK1 data in $\mathbf{c}$ and S4a. $n=3-5$. e Quantification of RIPK1 and SQSTM1 data in c. At day 1 after SCI $47.8 \%$ of RIPK1 positive cells were also positive for p62/SQSTM1. $n=8-13$. All data are presented as mean \pm SE. ${ }^{*} p<0.05,{ }^{* *} p<0.01,{ }^{* * *} p<0.001$

ribosomal protein S6, in the spinal cord tissue after SCI. Phospho-S6 was decreased in rapamycin-treated mice, confirming inhibition of mTOR (Fig. 6e, $\mathrm{f}$ and Supplementary Figure S8a). This was accompanied by decreased cleavage of $\alpha$-fodrin in rapamycin-treated as compared to vehicle treated SCI mice, confirming previously reported decreased cell damage and death (Fig. 6e, g and Supplementary Figure S8b $)^{21,24}$. Importantly, rapamycin treatment attenuated accumulation of p62/SQSTM1 at $24 \mathrm{~h}$ after SCI, indicating improvement in lysosomal function and autophagy flux (Fig. 6e, h and Supplementary Figure S8c). Consistent with the hypothesis that lysosomal dysfunction contributes to necroptosis, we also observed significant decrease in RIPK1 accumulation in rapamycintreated SCI animals as compared to vehicle controls (Fig. 6e, i and Supplementary Figure S8c). Rapamycin also attenuated accumulation of MLKL after SCI, although this effect failed to reach significance due to one outlier in the treated group (Fig. 6e, j and Supplementary Figure S8d).

In addition to being directly involved in necrosome formation, RIPK1 has been implicated in regulation of autocrine production of TNF $\alpha$ through the AKT/mTOR/ JNK pathway ${ }^{25,26}$. To determine if this pathway may also be affected, we assessed levels of AKT-Thr308 phosphorylation. Although we detected increase in phospho-AKT Thr308 after SCI, this was not altered by rapamycin treatment (Supplementary Figure S8a, e). Phosphorylation of JNK was also not affected by either SCI or rapamycin (Supplementary Figure S8b, f), suggesting that JNK pathway may not be essential for TNF $\alpha$ production at day 1 after SCI.

\section{Discussion}

We previously demonstrated that autophagy flux is temporarily inhibited following thoracic SCI in a rat model and is associated with enhancement of neuronal ER stress and caspase 12/caspase 3 dependent apoptosis ${ }^{12}$. Our current data confirm that autophagy is similarly inhibited after SCI in mice. The initial rapid increase in both p62/SQSTM1 and LC3-II and subsequent normalization of p62/SQSTM1 but not LC3-II levels also suggest that similarly to rat, autophagy flux is initially inhibited after mouse SCI but eventually recovers. At this point we are not certain whether eventual recovery of autophagy flux is due to death of the initially affected neuronal cells or if some of them are able to recover. However, our data indicate that the initial inhibition of autophagy flux is caused by a rapid decline in lysosomal function observed after SCI, as indicated by decrease in both the protein 


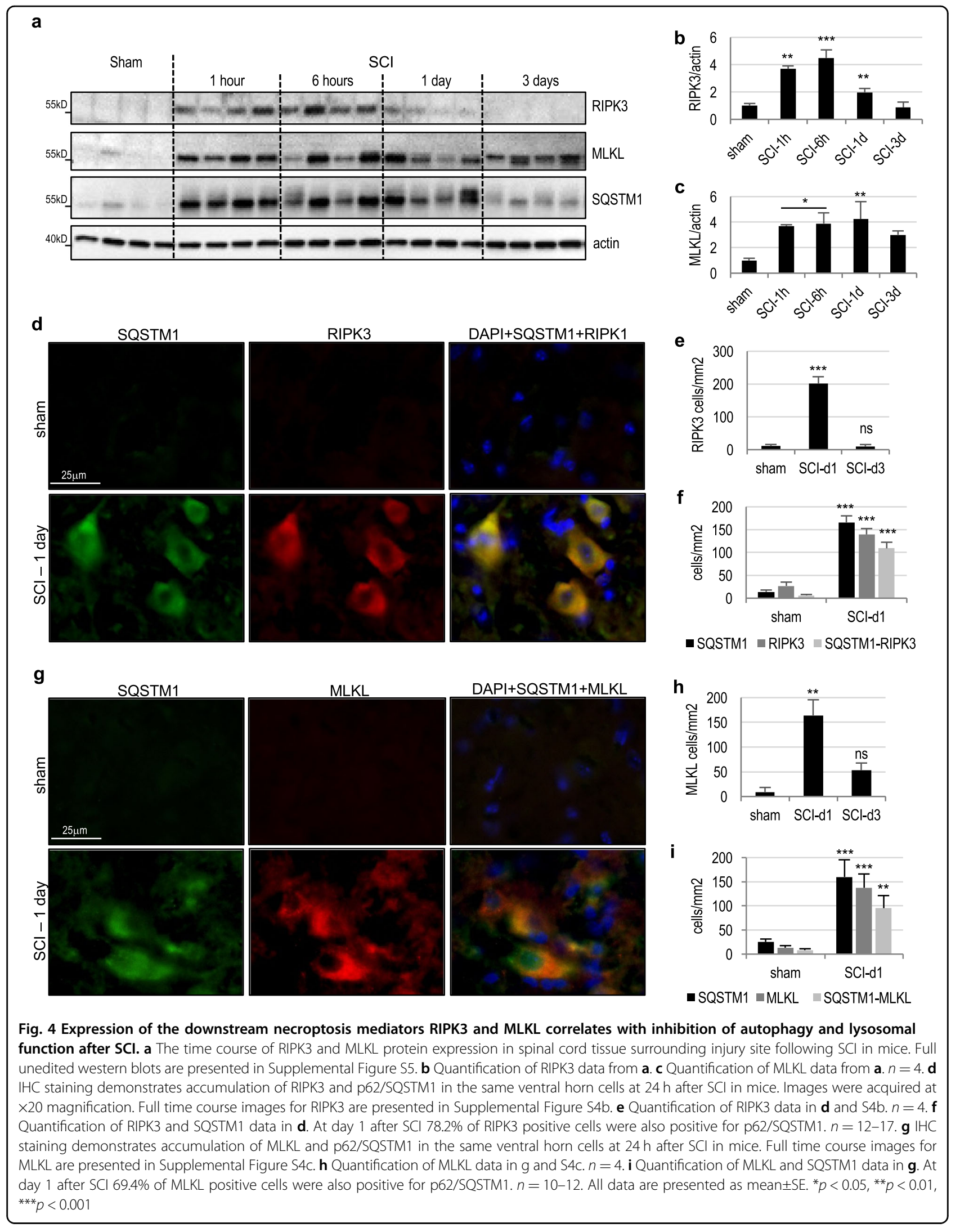




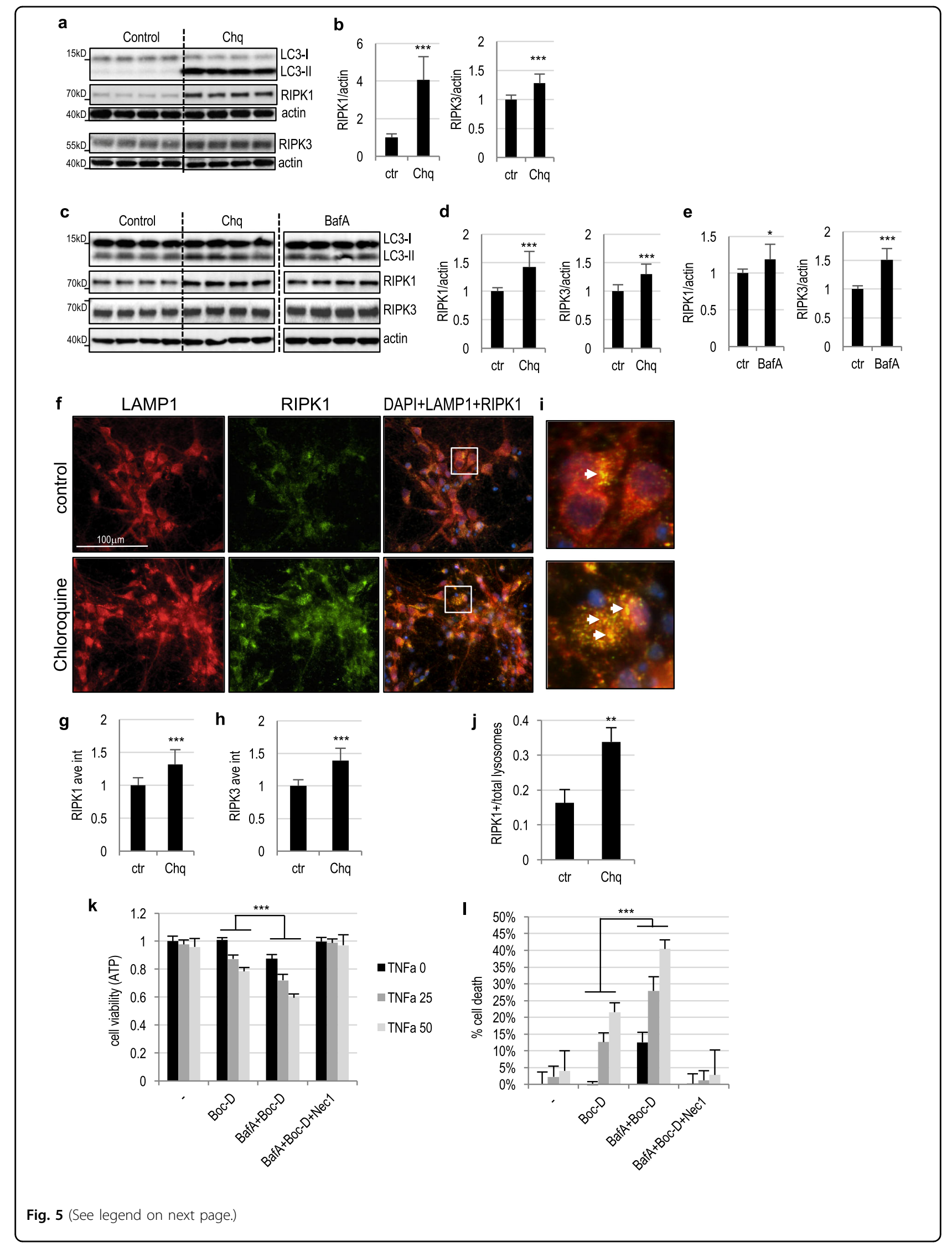




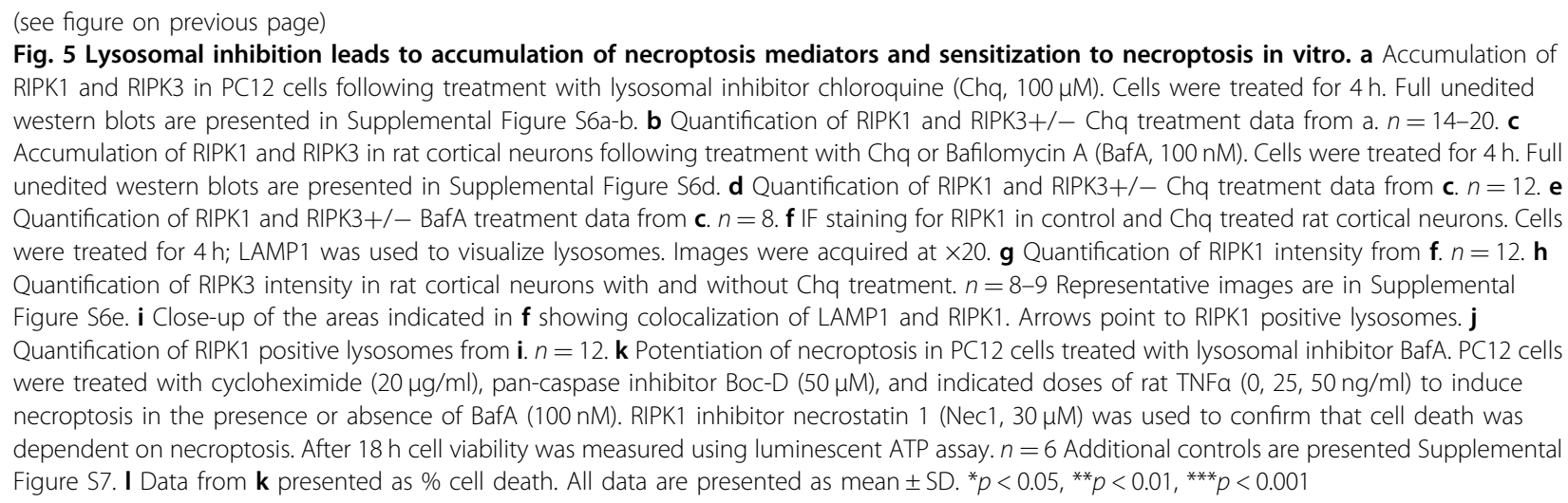

levels and activity of lysosomal enzymes. The timing of the lysosomal dysfunction is very rapid-it is apparent as early as $1 \mathrm{~h}$ after injury. This suggests that lysosomal damage may be one of the apical cellular events contributing to initiation, early propagation and potentiation of the secondary injury cascades.

Consistent with the notion that lysosomal damage may specifically contribute to multiple mechanisms of neuronal cell death after SCI, in addition to the previously reported ER stress and apoptosis ${ }^{12}$, we observed accumulation of markers of necroptosis specifically in the neurons displaying signs of autophagy flux inhibition and lysosomal damage. Interestingly, these markers, RIPK1, RIPK3, and MLKL are expressed after SCI with different kinetics. Although accumulation of each protein starts within hours after injury, RIPK1 continues to increase over the period of a week, before gradually subsiding. This time course likely reflects RIPK1 involvement in both cell death and inflammatory processes ${ }^{5}$. The latter are at least in part mediated through complex I/RIPK1 dependent activation of NFkB downstream of TNFR1. Consistent with this notion, while the earliest (day 1) expression of RIPK1 occurred primarily in neurons, at later time points it localized to cells with activated microglia/macrophage morphology (data not shown). On the other hand, while RIPK3 and MLKL are required together with RIPK1 for necrosome formation and induction of necroptosis ${ }^{19}$, they are not involved in complex I/RIPK1 dependent NFKB signaling. Consistent with a function in neuronal necroptosis after SCI, both RIPK3 and MLKL accumulate rapidly in neurons and peak by day 1 , the time of maximum cell death. Their levels decline by day 3 . This is also consistent with the timing of lysosomal and autophagy dysfunction after SCI, which declines by day 3 and largely resolves within a week after injury.

It has been previously demonstrated that activation of necroptosis contributes to neuronal and glial cell death after $\mathrm{SCI}^{8-10}$ and that treatment with RIPK1 kinase inhibitor, necrostatin 1 (nec1), can improve cell survival and functional outcomes after injury ${ }^{10,11}$. However, the mechanisms leading to activation of necroptosis after SCI and its relationship to other cellular pathways are not clear. In particular, while accumulation of RIPK1, RIPK3, and MLKL after SCI has been noted and used as a marker of necroptosis ${ }^{8}$, the reasons and timing for the buildup of these proteins remained unknown. Necroptosis is activated following ligation of the TNFR 1 receptor by TNF $\alpha$ and requires activation of RIPK1 and RIPK3 kinase activity and recruitment of MLKL to form necrosome $e^{5,19}$. No protein synthesis is necessary, and in fact, in many cell types necroptosis is specifically induced under conditions where protein translation is inhibited in order to suppress pro-survival function of $\mathrm{NFK}^{3} \mathrm{~B}^{3}$. Instead, RIPK1 protein recruitment to complex I versus complex II and necrosome is regulated by ubiquitination ${ }^{5}$. Ubiquitination also affects RIPK1 protein stability, which until recently has been thought to depend exclusively on proteasomal degradation. However, a recent report indicated that under at least some circumstances ubiquitinated RIPK1 and RIPK3 may be also degraded via lysosome-dependent pathway ${ }^{20}$. Consistent with the importance of this pathway in the CNS, our data suggest that lysosomal inhibition in vitro and in vivo specifically causes rapid accumulation of necroptosis mediators in neurons. Confirming the function of lysosomes in their degradation, RIPK1, RIPK3, and MLKL accumulate both in the cytosol and at the lysosomes. Therefore, our data support a model where under basal conditions lysosomal degradation keeps the levels of necroptosis mediators in neuronal cells low. When lysosomal function is impaired, such as after SCI, RIPK1, RIPK3, and MLKL accumulate, making the cells more sensitive to necroptosis.

Our in vitro experiments indicate that lysosomal inhibition can lead to induction of necroptosis even in the absence of TNF $\alpha$. Therefore, it is possible, that following lysosomal inhibition accumulation of RIPK1, RIPK3 and MLKL per se can lead to the formation of necrosome and initiation of necroptosis. It has been previously suggested 


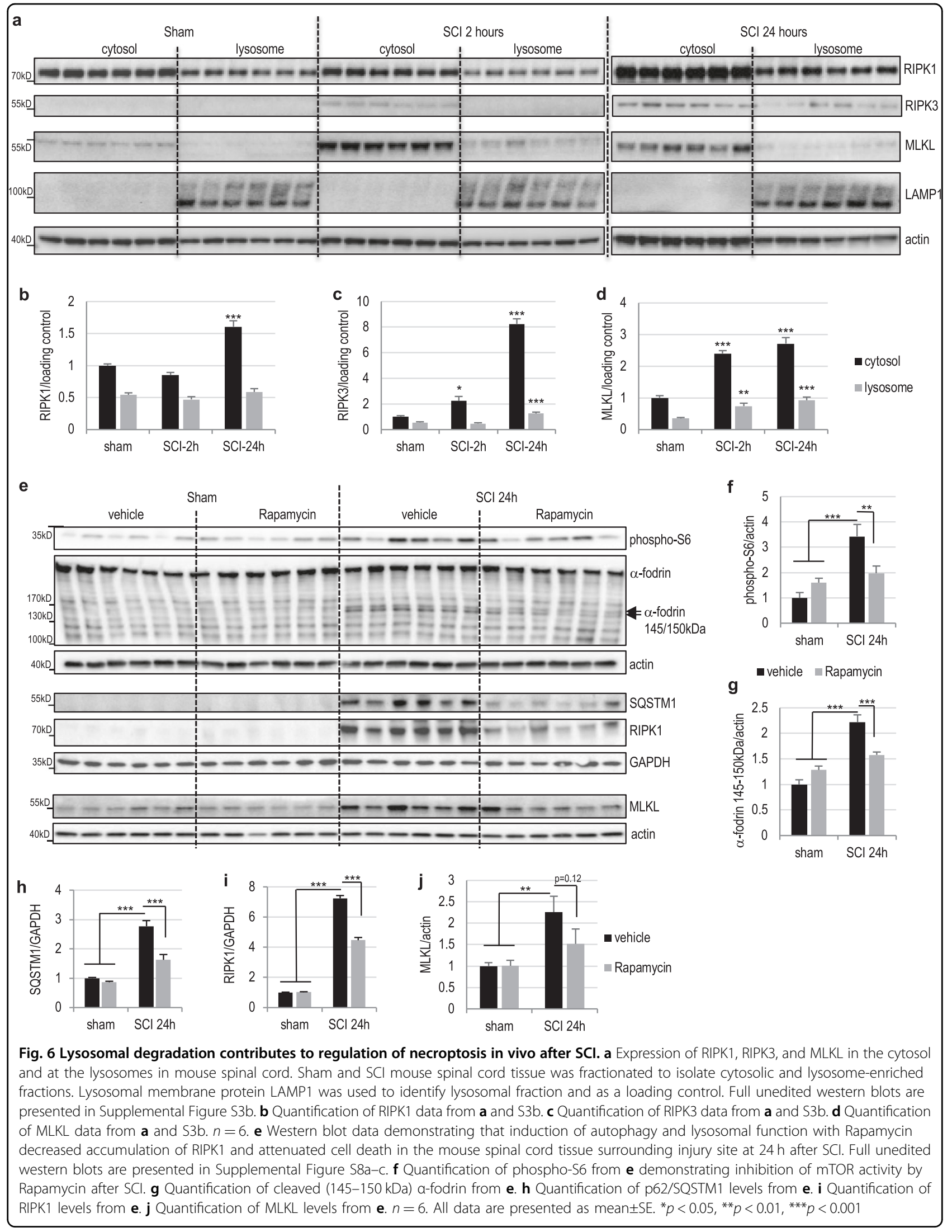


that under some circumstances formation of necrosomes can occur on the surface of dysfunctional autophagosomes through interaction with the autophagy protein, ATG $5^{27}$. However, we were not able to detect interaction of necrosome components with ATG5 either in vitro or in vivo (data not shown). Furthermore, our in vitro IF experiments indicated that RIPK1 colocalized primarily with LAMP1-positive lysosomes rather than with autophagosomes (data not shown). However, we cannot exclude the possibility that in addition to directly leading to accumulation of necroptosis proteins, lysosomal dysfunction after SCI can further potentiate necroptosis after SCI by causing accumulation of autophagosomes and thus providing additional platform for necrosome assembly. These mechanisms are not mutually exclusive and further experiments will be necessary to clarify their respective contribution.

Our data point to the crucial role of lysosomal dysfunction as an early event contributing to propagation of secondary injury through induction of apoptosis and necroptosis. Consistently with the causative role of lysosomal dysfunction, treatment of SCI mice with the mTOR inhibitor and inducer of autophagy and lysosomal biogenesis, rapamycin, attenuated both inhibition of autophagy flux and accumulation of RIPK1. Treatment with rapamycin has been previously shown to confer neuroprotection and improve functional outcomes in rodent models of $\mathrm{SCI}^{21,22,28}$. However, the mechanisms of this protection remained unclear. Interestingly, combined inhibition of mTOR and AKT but not either treatment alone, has been shown to suppress necroptosis in cultured cortical neurons ${ }^{29}$ and provide neuroprotection in a mouse model of traumatic brain injury (TBI) ${ }^{23}$. Our data indicate that inhibition of mTOR is sufficient to at least partially attenuate necroptosis after SCI. In addition to being directly involved in necrosome formation, RIPK1 has been implicated in regulation of autocrine production of TNF $\alpha$ through the Akt/mTOR/JNK pathway ${ }^{25,26}$. We did not observe any changes in either AKT or JNK activation following treatment with only rapamycin. Therefore, similar to TBI, inhibition of both AKT and mTOR may be necessary to cause downregulation of JNK and autocrine production of TNF $\alpha$. We hypothesize that combination of Akt inhibitor and rapamycin treatment after neurotrauma may affect necroptosis by both suppressing accumulation of RIPK1 and other necrosome components, and by decreasing autocrine TNF $\alpha$ production and activation of necrosome assembly downstream of TNFR1 ligation.

Together our data suggest that, lysosomal function may be an important upstream cellular event involved in control of multiple pro-death pathways during SCI secondary injury, including inhibition of autophagy flux, activation of ER stress dependent apoptosis and sensitization to necroptosis. Thus, restoring or enhancing lysosomal function may represent an interesting intervention avenue ${ }^{28}$. Inhibition of autophagy/lysosomal pathway and activation of necroptosis have been shown to contribute to cell loss and tissue damage in other CNS trauma models, including traumatic brain injury ${ }^{16,23,30,31}$. Necroptosis is also involved in neurodegenerative diseases affecting the spinal cord, such as amyotrophic lateral sclerosis and multiple sclerosis ${ }^{6,7}$. As most neurodegenerative disorders, these diseases are also associated with defects in the lysosomal/autophagy pathway. Therefore, lysosomal dysfunction may contribute to induction and/ or sensitization to necroptosis also in these diseases.

\section{Materials and methods \\ Contusive $\mathrm{SCl}$ in mice}

Adult male C57BL/6J mice (8-10 weeks old, $20-25$ g; Jackson Laboratory) or GFP-LC3 autophagy reporter transgenic male mice ${ }^{15}$ were anesthetized with isoflurane and received T10 spinal contusions using the Infinite Horizon Spinal Cord Impactor (Precision Systems and Instrumentation) with a force of $60 \mathrm{kdyn}$, a moderate injury $^{13,32}$. Bladders were manually expressed twice daily until a reflex bladder was established (7-14 d after SCI). The number of mice at various time points in each study is indicated in the figure legends. All procedures were performed under protocols approved by the University of Maryland School of Medicine Institutional Animal Care and Use Committee (IACUC).

\section{Drug treatments}

Sham and SCI mice were assigned to a treatment group according to a randomized block experimental design. Rapamycin (Sigma-Aldrich, US, Cat. No. 37094) was dissolved in DMSO and then diluted in vehicle containing $0.25 \%$ PEG400 and $0.25 \%$ Tween 80 . The final concentration of DMSO was adjusted to $0.1 \%$. Rapamycin was injected twice ( $5 \mathrm{~min}$ and $4 \mathrm{~h}$ post injury) intraperitoneally in treatment group $(n=6)$ at a dose of $5 \mathrm{mg} / \mathrm{kg}$ based on prior investigation ${ }^{16}$. Mice of the control group $(n=6)$ were injected with the equivalent volume of vehicle. Animals were anesthetized and processed for western blot at $24 \mathrm{~h}$ after injury.

\section{Western blot analysis}

Mouse spinal cord tissue $(5 \mathrm{~mm})$ centered on the injury site was lysed and homogenized in RIPA buffer (SigmaAldrich) supplemented with $1 \times$ protease inhibitor cocktail (Sigma-Aldrich) and phosphatase inhibitor cocktail II and III (Sigma-Aldrich), sonicated and centrifuged at $20,000 \times g$ for $20 \mathrm{~min}^{12,32}$. Protein concentration was determined by the Pierce BCA method (ThermoFisher Scientific, US). Samples were run on 4-20\% SDS-PAGE (Bio-Rad, Hercules, CA), and transferred to PVDF 
membrane (Bio-Rad). Membranes were incubated with primary antibodies overnight, with HRP-conjugated secondary antibodies for $1 \mathrm{~h}$, visualized using SuperSignal West Dura Extended Duration Substrate (ThermoFisher), and imaged with ChemIDoc TM MP system (Bio-Rad). The signal (optical density) was quantified by Image Lab software (Bio-Rad). Primary antibodies: LC3 (1:1000, Novus, Cat. No. NB100-2220), p62/SQSTM1 (1:1000; BD Bioscience, Cat. No. 610832), CTSD (1:100, Santa Cruz Biotechnology, Cat. No. sc-6486), RIPK1 (1:1000; Cell Signaling, Cat. No. 3493, Danvers, MA), RIPK3 (1:1000, ProSci, Cat. No. 2283, Poway, CA), MLKL (1:1000, EMD Millipore, Cat. No. MABC604, Temecula, CA), $\beta$-Actin (1:10 000; Sigma-Aldrich, Cat. No. A1978), Fordin/ SPTAN1 (1:5000; Enzo Life Science International, Cat. No. BML-FG6090), GAPDH (1:2000; Millipore, Cat. No. AB2302), LAMP1 (1:1000; Cat. No. 1D4B, developed by J. Thomas August, Developmental Studies Hybridoma Bank/NICHD, maintained by The University of Iowa, Department of Biology, Iowa City, IA). Expression levels of the target protein bands were normalized to $\beta$-actin, GAPDH or LAMP1 (lysosomal fraction), and expressed as a fold of sham control.

\section{Subcellular fractionation}

Around 5-mm fragments of spinal cord tissue centered on the injury site or corresponding site in sham animals were collected from sham mice, and at 2 or $24 \mathrm{~h}$ after injury and homogenized in ice-cold buffered solution containing $0.32 \mathrm{M}$ Sucrose, $10 \mathrm{mM}$ Hepes and protease and phosphatase inhibitors. Homogenates were centrifuged at $800 \times g$ for $10 \mathrm{~min}$ at $4{ }^{\circ} \mathrm{C}$ to pellet down the nuclei. Supernatants were sequentially centrifuged at $20,000 \times g$ for $20 \mathrm{~min}$ at $4{ }^{\circ} \mathrm{C}$ to pellet the heavy membrane/crude lysosomal fractions and at 100,000 $\times g$ for $1 \mathrm{~h}$ at $4{ }^{\circ} \mathrm{C}$ to pellet light membrane fractions ${ }^{16}$. Both supernatant and suspended pellet fractions were re-centrifuged to minimize cross contamination from the different subcellular fractions. All pellets were re-suspended in homogenization buffer. Protein concentration was estimated using $\mathrm{BCA}$ reagent; samples were analyzed by western blot.

\section{Lysosomal activity assays}

Mice were anesthetized, perfused with ice-cold saline, and 5-mm spinal cord tissue surrounding the site of injury was dissected, homogenized in ice cold cell lysis buffer and centrifuged at $15,000 \times g$ for $5 \mathrm{~min}$ at $4{ }^{\circ} \mathrm{C}$. Protein concentration was estimated by the BCA method; $50 \mathrm{ng}$ of protein was used per assay. The CTSD and NAG assays were performed using a fluorometric assay kits (Abcam, Cat. No. ab65302, Sigma-Aldrich, Cat. No. CS0780) as per the manufacturer's instructions ${ }^{16}$. Fluorescence released from the synthetic substrate was measured using fluorescent plate reader (Synergy Hybrid, Biotek) at Ex/ $\mathrm{Em}=328 / 460 \mathrm{~nm}$.

\section{Immunohistochemistry (IHC)}

Animals were intracardially perfused with $\mathrm{PBS}$, then with $4 \%$ paraformaldehyde ${ }^{32,33}$. A $1.0-\mathrm{cm}$ segment of spinal cord centered at the injury epicenter was sectioned at $20 \mu \mathrm{m}$ thickness and thaw-mounted onto Superfrost Plus slides (ThermoFisher). Sections were blocked in 5\% goat or donkey serum in PBS/0.025\% Triton X-100, incubated with primary antibodies overnight and with secondary antibodies for $1 \mathrm{~h}$. Cell nuclei were labeled with 4',6-diamidino-2-phenylindole (DAPI, Sigma-Aldrich), slides were cover-slipped with an anti-fading medium (Hydromount; National Diagnostics). Primary antibodies: LC3 (1:200, Novus, Cat. No. NB100-2220), p62/SQSTM1 (1:200; Progen, Cat. No. GP62-C, Heidelberg, Germany), CTSD (1:100, Santa Cruz Biotechnology, Cat. No. sc6486), RIPK1 (1:100; Cell Signaling, Cat. No. 3493, Danvers, MA), RIPK3 (1:100, Enzo, Cat.No. ADI 905-242-100, Farmingdale, NY), MLKL (1:100, EMD Millipore, Cat. No. MABC604, Temecula, CA), NeuN/RBFOX3 (1:500; Millipore, Cat. No. MAB377). Secondary antibodies: alexa fluor 488 goat anti-rabbit (Cat. No. A11034), alexa fluor 546 goat anti-mouse (Cat. No. A11030), alexa fluor 568 goat anti-guinea pig (Cat. No. A11075), alexa fluor 633 goat anti-mouse (Cat. No. A21052) and alexa fluor 546 donkey anti-goat (Cat. No. A11056, all Invitrogen).

\section{Image acquisition and quantification}

All images were acquired $0.5-1 \mathrm{~mm}$ rostral to the epicenter $^{33}$. Images from ventral horns of gray matter were acquired using a fluorescent Nikon Ti-E inverted or Nikon Ni-E upright microscope, at $\times 20$ (CFI Plan APO VC $20 \times$ NA 0.75 WD $1 \mathrm{~mm}$ ) or $\times 60$ (CFI Plan APO VC $60 \times$ NA 1.4 Oil) magnification ${ }^{12,16}$. All images for each data set were acquired using the same parameters (magnification, exposure time, gain, etc). All $\times 60$ images were acquired as z-stacks and focused using Extended Depth of Focus (EDF) module of Elements software (Nikon). The background of each image was subtracted using background region of interest (ROI). All images were quantified in unbiased automated manner using custom macros in Elements: nuclei were identified using Spot Detection algorithm; cells positive for any of the immunofluorescence markers were identified using Detect Regional Maxima algorithm, followed by global thresholding. Number of positive cells was normalized to the total imaged ventral horn area $\left(\mathrm{mm}^{2}\right)$. Intracellular puncta were detected using Spot Detection and normalized to the number of cells imaged. For each experiment data from all images from same region in each mouse was summed up and used for final statistical analysis. At least $500-1000$ cells were quantified per mouse per experiment. All 
quantification was performed on original unedited images. For visualization purposes in figures only brightness and contrast were adjusted; all adjustments were applied to entire image area and equally to all panels in the same figure. In multi-color overlay images brightness of the DAPI channel was selectively decreased to allow better visualization of other channels.

\section{Tissue culture and treatments}

Rat pheochromocytoma PC12 cells (ATCC, Cat. No. CRL-1721.1) were maintained in F-12K nutrient mixture $(1 \times)$ Kaighn's modification with $5 \%$ fetal bovine serum (FBS, Gibco, Cat. No.10082-147), 10\% horse serum (Gibco), penicillin, and streptomycin ${ }^{34}$. Rat embryonic neurons were extracted from embryo of female spraquedawley rats (E17, from Taconic) as described ${ }^{35}$. Neurons were maintained in neurobasal medium (Gibco, Cat. No. 12348-017) with B-27, L-glutamine, penicillin and streptomycin and allowed to differentiate for 1 week prior to experiments. For western blot analysis, PC12 cells were seeded in 24-well plates at a density of $5 \times 10^{4}$ cells/well. After reaching $80 \%$ confluence (PC12 cells) or 1 week differentiation (neurons), cells were incubated with or without $100 \mu \mathrm{M}$ chloroquine (Sigma-Aldrich, Cat. No. C6628) or $100 \mathrm{nM}$ bafilomycin A (Sigma-Aldrich, Cat. No. B1793) for $4 h^{14}$, lysed in homogenizing buffer and analyzed by western blot. For immunofluorescence (IF) imaging cells were seeded on cover slips, treated as above, then fixed in $2 \%$ paraformaldehyde. Coverslips were blocked in $5 \%$ goat or donkey serum in PBS/0.025\% Triton X-100, incubated with primary antibodies (same as for IHC) overnight and with secondary antibodies for $1 \mathrm{~h}$. Cell nuclei were labeled with DAPI. Coverslips were mounted on slides with an anti-fading medium (Hydromount; National Diagnostics, Atlanta, GA), then imaged and analyzed as IHC above ${ }^{12}$. For cell viability assay, PC12 cells were seeded in 96-well plates at a density of $5 \times 10^{3}$ cells/well. After reaching $80 \%$ confluence, cells were incubated with or without $50 \mu \mathrm{M}$ Boc-D-FMK (Cayman Chemical Company, Cat. No. 16118), $30 \mathrm{mM}$ necrostatin1 (Cayman Chemical Company, Cat. No. 11658) ${ }^{3}, 20 \mu \mathrm{g} /$ $\mathrm{ml}$ cycloheximide (Sigma-Aldrich, Cat. No. C7698), 100 $\mu \mathrm{M}$ chloroquine, $100 \mathrm{nM}$ bafilomycin $\mathrm{A}$, and recombinant rat TNF $\alpha(0,25,50 \mathrm{ng} / \mathrm{ml}$ BioLegend, Cat. No. 580102) for $18 \mathrm{~h}$. Cell viability was analyzed by CellTiter-Glo Luminescnet Cell Viability Assay kit (Promega, Cat. No. G7570) following the manufacturer protocol ${ }^{36}$.

\section{Statistical analysis}

Unless indicated otherwise, all in vivo results are expressed as mean \pm SEM, where " $n$ " is the number of individual animals per group. The number of animals in all studies was determined by power analysis (power of 0.8 with alpha value 0.05 ). Key experiments were repeated with independent groups of animals to ensure reproducibility. For in vitro cell based assays results are expressed as mean $\pm \mathrm{SD}$; " $n$ " is the number of total replicates from at least three independent experiments. All statistical analyses were conducted using SigmaPlot, Version 12 (Systat Software, San Jose, CA) or GraphPad Prism, Version 3.02 for Windows (GraphPad Software, La Jolla, CA). One-way or two-way (cell death assay and in vivo rapamycin treatment) ANOVA followed by Bonferroni, Tukey's or SNK $t$-test post-hoc test was used for parametric data. Kruskal-Wallis ANOVA based on ranks and Dunn's post-hoc test was used for non-parametric data. For experiments with only two groups two-tailed unpaired Student's $t$-test (parametric) was performed. A $p$ value $\leq$ 0.05 was considered significant ${ }^{12,16}$.

\section{Acknowledgements}

We thank Dr. Noboru Mizushima (Tokyo Medical and Dental University, Tokyo, Japan) and Dr. Beth Levine (UT Southwestern Medical center, Dallas TX) for the GFP-LC3 mice, Ms. Jingwen Yu and Lulu Liu for expert technical support. This study was supported by the National Institutes of Health Grants R01 NS094527 to J.W. and M.M.L., R01 NS091218 to M.M.L., and 2R01 NR013601 to J.W.

\section{Author details}

${ }^{1}$ Department of Anesthesiology and the Center for Shock, Trauma and Anesthesiology Research (STAR), University of Maryland School of Medicine, Baltimore, MD, USA. ${ }^{2}$ Department of Orthopaedics, University of Maryland School of Medicine, Baltimore, MD, USA

\section{Authors' contributions}

M.M.L., J.W., and E.Y.K. conceived the project; M.M.L., J.W., S.L., E.Y.K., and C.S. designed experiments; S.L., Y.L., H.C., C.S., and J.W. performed experiments; M. M.L., S.L., Y.L., H.C., and J.W. analyzed data and prepared figures; M.M.L. and J.W. wrote the manuscript with input from all the authors.

Conflict of interest

The authors declare that they have no conflict of interest.

\section{Publisher's note}

Springer Nature remains neutral with regard to jurisdictional claims in published maps and institutional affiliations.

Supplementary Information accompanies this paper at https://doi.org/ 10.1038/s41419-018-0469-1.

Received: 27 December 2017 Revised: 27 February 2018 Accepted: 5 March 2018

Published online: 23 April 2018

\section{References}

1. Beattie, M. S., Hermann, G. E., Rogers, R. C. \& Bresnahan, J. C. Cell death in models of spinal cord injury. Prog. Brain Res. 137, 37-47 (2002).

2. Faden, A. I. \& Stoica, B. Neuroprotection: challenges and opportunities. Arch. Neurol. 64, 794-800 (2007).

3. Degterev, A. et al. Chemical inhibitor of non-apoptotic cell death with therapeutic potential for ischemic brain injury. Nat. Chem. Biol. 1, 112-119 (2005).

4. Vandenabeele, P., Galluzzi, L., Vanden Berghe, T. \& Kroemer, G. Molecular mechanisms of necroptosis: an ordered cellular explosion. Nat. Rev. Mol. Cell Biol. 11, 700-714 (2010).

5. Ofengeim, D. \& Yuan, J. Regulation of RIP1 kinase signalling at the crossroads of inflammation and cell death. Nat. Rev. Mol. Cell Biol. 14, 727-736 (2013). 
6. Ito, Y. et al. RIPK1 mediates axonal degeneration by promoting inflammation and necroptosis in ALS. Science 353, 603-608 (2016).

7. Ofengeim, D. et al. Activation of necroptosis in multiple sclerosis. Cell Rep. 10 1836-1849 (2015).

8. Fan, $\mathrm{H}$. et al. Involvement of endoplasmic reticulum stress in the necroptosis of microglia/macrophages after spinal cord injury. Neuroscience 311, 362-373 (2015).

9. Fan, $\mathrm{H}$. et al. Reactive astrocytes undergo M1 microglia/macrohpages-induced necroptosis in spinal cord injury. Mol. Neurodegener. 11, 14 (2016).

10. Liu, M. et al. Necroptosis, a novel type of programmed cell death, contributes to early neural cells damage after spinal cord injury in adult mice. J. Spinal Cord. Med. 38, 745-753 (2015).

11. Wang, Y. et al. Necroptosis inhibitor necrostatin-1 promotes cell protection and physiological function in traumatic spinal cord injury. Neuroscience $\mathbf{2 6 6}$, 91-101 (2014)

12. Liu, S. et al. Disrupted autophagy after spinal cord injury is associated with ER stress and neuronal cell death. Cell Death Dis. 6, e1582 (2015).

13. Wu, J. et al. Spinal cord injury causes brain inflammation associated with cognitive and affective changes: role of cell cycle pathways. J. Neurosci.: Off. J. Soc. Neurosci. 34, 10989-11006 (2014).

14. Klionsky, D. J. et al. Guidelines for the use and interpretation of assays for monitoring autophagy (3rd edition). Autophagy 12, 1-222 (2016).

15. Mizushima, N., Yamamoto, A., Matsui, M., Yoshimori, T. \& Ohsumi, Y. In vivo analysis of autophagy in response to nutrient starvation using transgenic mice expressing a fluorescent autophagosome marker. Mol. Biol. Cell 15, 1101-1111 (2004).

16. Sarkar, S. et al. Impaired autophagy flux is associated with neuronal cell death after TBI. Autophagy 10, 0 (2014).

17. Moon, C. et al. Immunohistochemical study of cathepsin D in the spinal cords of rats with clip compression injury. J. Vet. Med Sci. 70, 937-941 (2008).

18. $\mathrm{Wu}$, J. et al. Interaction of $\mathrm{NG2}(+)$ glial progenitors and microglia/macrophages from the injured spinal cord. GLIA 58, 410-422 (2010).

19. Wang, $\mathrm{H}$. et al. Mixed lineage kinase domain-like protein MLKL causes necrotic membrane disruption upon phosphorylation by RIP3. Mol. Cell 54, 133-146 (2014).

20. Seo, J. et al. CHIP controls necroptosis through ubiquitylation- and lysosomedependent degradation of RIPK3. Nat. Cell Biol. 18, 291-302 (2016).

21. Sekiguchi, A., Kanno, H., Ozawa, H., Yamaya, S. \& Itoi, E. Rapamycin promotes autophagy and reduces neural tissue damage and locomotor impairment after spinal cord injury in mice. J. Neurotrauma 29, 946-956 (2012)
22. Wang, Z. Y., Liu, W. G., Muharram, A., Wu, Z. Y. \& Lin, J. H. Neuroprotective effects of autophagy induced by rapamycin in rat acute spinal cord injury model. Neuroimmunomodulation 21, 257-267 (2014).

23. Park, J. et al. Combination therapy targeting Akt and mammalian target of rapamycin improves functional outcome after controlled cortical impact in mice. J. Cereb. Blood Flow. Metab. 32, 330-340 (2012).

24. Goldshmit, Y. et al. Rapamycin increases neuronal survival, reduces inflammation and astrocyte proliferation after spinal cord injury. Mol. Cell. Neurosci. 68, 82-91 (2015).

25. Christofferson, D. E. et al. A novel role for RIP1 kinase in mediating TNFalpha production. Cell Death Dis. 3, e320 (2012).

26. McNamara, C. R. et al. Akt Regulates TNFalpha synthesis downstream of RIP1 kinase activation during necroptosis. PLOS ONE 8, e56576 (2013).

27. Basit, F., Cristofanon, S. \& Fulda, S. Obatoclax (GX15-070) triggers necroptosis by promoting the assembly of the necrosome on autophagosomal membranes. Cell Death Differ. 20, 1161-1173 (2013).

28. Lipinski, M. M. \& Wu, J. Modification of autophagy-lysosomal pathway as a neuroprotective treatment for spinal cord injury. Neural Regen. Res. 10, 892-893 (2015).

29. Liu, Q. et al. Akt and mTOR mediate programmed necrosis in neurons. Cell Death Dis. 5, e1084 (2014).

30. You, Z. et al. Necrostatin-1 reduces histopathology and improves functional outcome after controlled cortical impact in mice. J. Cereb. Blood Flow. Metab. 28, 1564-1573 (2008)

31. Lipinski, M. M., Wu, J., Faden, A. I. \& Sarkar, C. Function and mechanisms of autophagy in brain and spinal cord trauma. Antioxid. Redox Signal 23, 565-577 (2015).

32. $\mathrm{Wu}, \mathrm{J}$. et al. Endoplasmic reticulum stress and disrupted neurogenesis in the brain are associated with cognitive impairment and depressive-like behavior after spinal cord injury. J. Neurotrauma 33, 1919-1935 (2016).

33. Wu, J. et al. Ablation of the transcription factors E2F1-2 limits neuroinflammation and associated neurological deficits after contusive spinal cord injury. Cell Cycle 14, 3698-3712 (2015).

34. Smith, W. W. et al. Endoplasmic reticulum stress and mitochondrial cell death pathways mediate A53T mutant alpha-synuclein-induced toxicity. Hum. Mol. Genet 14, 3801-3811 (2005).

35. Ofengeim, D. et al. Identification of small molecule inhibitors of neurite loss induced by Abeta peptide using high content screening. J. Biol. Chem. 287, 8714-8723 (2012).

36. Hitomi, J. et al. Identification of a molecular signaling network that regulates a cellular necrotic cell death pathway. Cell 135, 1311-1323 (2008). 